\title{
The Malaysian Cataract Surgery Registry: risk Indicators for posterior capsular rupture
}

\author{
Mohamad Aziz Salowi, ${ }^{1,2}$ Fiona L M Chew, ${ }^{3}$ Tassha Hilda Adnan, ${ }^{4}$ Christopher King, ${ }^{5}$ \\ Mariam Ismail, ${ }^{6}$ Pik-Pin Goh ${ }^{7}$
}

${ }^{1}$ Faculty of Medicine, Universiti Sultan Zainal Abidin, Kuala Terengganu, Malaysia 2Department of Ophthalmology, Hospital Selayang, Batu Caves, Malaysia

${ }^{3}$ Department of Ophthalmology, Kuala Lumpur Hospital, Kuala Lumpur, Malaysia ${ }^{4}$ Biostatistics Unit, National Clinical Research Centre, Kuala Lumpur, Malaysia

${ }^{5}$ Buckinghamshire Healthcare NHS Trust, UK

${ }^{6}$ Department of Ophthalmology, Sultan Abdul Halim Hospital, Sungai Petani, Kedah, Malaysia ${ }^{7}$ Clinical Research Centre, National Clinical Research Centre, Kuala Lumpur, Malaysia

Correspondence to

Dr Fiona L M Chew, Department of Ophthalmology, Kuala Lumpur Hospital, Department of Ophthalmology, Kuala Lumpur Hospital, Jalan Pahang, Kuala Lumpur, 50586 Malaysia; sabrefmin@gmail.com

Received 8 November 2016 Revised 8 February 2017 Accepted 18 February 2017 Published Online First 6 July 2017

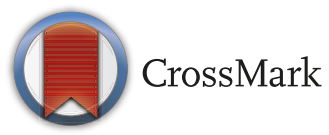

To cite: Salowi MA, Chew FLM, Adnan TH, et al. Br J Ophthalmol 2017:101:1466-1470.

\section{ABSTRACT}

Aim To identify the risk indicators for posterior capsular rupture $(P C R)$ in the Malaysian Cataract Surgery Registry (CSR).

Methods Data from the web-based CSR were collected for cataract surgery performed from 2008 to 2013. Data was contributed by 36 Malaysian Ministry of Health public hospitals. Information on patient's age, ethnicity, cause of cataract, ocular and systemic comorbidity, type of cataract surgery performed, local anaesthesia and surgeon's status was noted. Combined procedures and type of hospital admission were recorded. PCR risk indicators were identified using logistic regression analysis to produce adjusted OR for the variables of interest.

Results A total of 150213 cataract operations were registered with an overall PCR rate of $3.2 \%$. Risk indicators for PCR from multiple logistic regression were advancing age, male gender $(95 \% \mathrm{Cl} 1.04$ to $1.17 ; \mathrm{OR}$ 1.11), pseudoexfoliation $(95 \% \mathrm{Cl} 1.02$ to $1.82 ; \mathrm{OR}$ 1.36), phacomorphic lens $(95 \% \mathrm{Cl} 1.25$ to 3.06 ; OR 1.96), diabetes mellitus ( $95 \% \mathrm{Cl} 1.13$ to 1.29 ; OR 1.20) and renal failure (95\% Cl 1.09 to 1.55; OR 1.30). Surgical PCR risk factors were combined vitreoretinal surgery $(95 \% \mathrm{Cl} 2.29$ to 3.63; OR 2.88) and less experienced cataract surgeons. Extracapsular cataract extraction $(95 \% \mathrm{Cl} 0.76$ to 0.91 ; OR 0.83$)$ and kinetic anaesthesia were associated with lower PCR rates. Conclusions This study was agreed with other studies for the risk factors of PCR with the exception of local anaesthesia given and type of cataract surgery. Better identification of high-risk patients for PCR decreases intraoperative complications and improves cataract surgical outcomes.

\section{INTRODUCTION}

Posterior capsular rupture (PCR) is a dreaded complication of cataract surgery that significantly impacts the patient's postoperative visual outcome and cost of cataract surgery. PCR is used as the main parameter for cataract surgery competency and is measured via various methods such as surgical logbook review, case-mix assessment, cumulative summation technique and various risk scoring systems. ${ }^{1-5}$ The common reported risks for PCR include cataract morphology such as traumatic and high-density cataract and ocular comorbidity like small pupil, pseudoexfoliation, glaucoma and previous vitrectomy. Other risks include patient factors such as age, ethnicity and the inability to lie flat. ${ }^{6-8}$

Identification of preoperative PCR risk is vital as it allows risk stratification for surgeons to enhance the preoperative counselling process, as well as take precautionary measures intraoperatively. This would also help with surgical case selection for ophthalmology trainees. This study aims to identify the risk factors of PCR in the Malaysian population based on 5-year data from the Malaysian Ministry of Health Cataract Surgery Registry (MOH CSR).

\section{MATERIALS AND METHODS}

Ethical approval was obtained from the Medical Research and Ethics Committee of the MOH. The study also conformed to all local laws and was compliant with the guidelines of the Declaration of Helsinki. Data were prospectively collected from the web-based CSR of the MOH. CSR is part of the National Eye Database, a web-based password protected surveillance system collecting data on eye diseases and clinical performance of the ophthalmology service in Malaysia. It consists of systematic data entry according to predefined sets of preoperative, operative and outcome forms by designated paramedical staff. $\mathrm{MOH}$ ophthalmology departments nationwide contribute data to the CSR database. Details on the Malaysian CSR have been published elsewhere. ${ }^{9} 10$

All consecutive patients who underwent cataract surgery from 1 January 2008 to 31 December 2013 were identified as potential study subjects. The patients were recruited as study subjects if they were aged 50 years and above and had undergone either phacoemulsification or extracapsular cataract extraction (ECCE) surgery. Patients were excluded if they had secondary intraocular lens implantation as the primary procedure. The factors of interest in this study were patient's demographic profile, ocular and systemic comorbidities, surgeon seniority and type of admission, if combination surgery was performed and the type of local anaesthesia used. All eyes were taken for analysis. Subjects with intraoperative PCR (PCR group) were compared with subjects who did not have PCR (control group) to look for risk factors. The subjects were further subdivided into four age categories which were the 50-59, 60-69, 70-79 years and above 80 years category.

Specialists were defined as surgeons who had obtained ophthalmic qualification and passed the probation period of 6-18 months. Gazetting specialists were defined as surgeons who had obtained ophthalmic qualification and were undergoing the probation period. Medical officers were defined as surgeons under training for ophthalmic qualification.

\section{Statistical methods}

Data collected were analysed using IBM SPSS Statistics 20 (IBM SPSS Statistics for Windows, 
IBM). Continuous variables were presented by mean and SD for normally distributed data and median and IQR for skewed data, and categorical data were presented by frequency and percentage. The simple logistic regression model was used to estimate the OR for age, gender, race, cause of cataract (primary and secondary), type of admission, type of surgery, type of anaesthesia, ocular comorbidity of the eye, surgeon status, systemic comorbidity and type of combined surgery. ORs indicating the effect of the risk factors on the occurrence of PCR during cataract surgery were calculated and reported with 95\% CIs. The backward stepwise model was then carried out in the multiple logistic regression including all the risk factors, except for secondary cause of cataract (trauma, drug induced and surgery induced) as the number of the observations was small. Adjusted OR and its $95 \%$ CI were used to estimate a risk score for combinations of risk factors. The $\mathrm{p}$ values $<0.05$ were considered statistically significant.

\section{RESULTS}

Of the 174674 cataract operations registered during the study period, 150213 eyes belonged to patients aged 50 years and above who underwent either phacoemulsification or ECCE. There was a steady increase in the number of cataract surgeries being documented throughout the years. The percentage of intraoperative complications in total was $6.1 \%$. The yearly PCR rate ranged from $2.7 \%$ to $3.7 \%$, with an overall PCR rate of $3.2 \%$. Both intraoperative complication and PCR rate showed a decreasing trend over the years. The more serious complications such as dropped nucleus and suprachoroidal haemorrhage were not frequent and the trend remained unchanged over time (table 1).

A total of 4654 eyes had documented intraoperative PCR. Most study subjects (80.4\%, $120790 / 150$ 213) were aged 60 years and above. The PCR group was noted to have an overall higher mean (67.8 years vs 67.2 years) and median age (68.0 years vs 67.0 years) in comparison to the study group $(p<0.001)$. The age groups showed that the $70-79$ and $\geq 80$ years old categories had a higher risk of PCR $(17 \%$ and $23 \%$ higher risk, respectively) compared with subjects aged 59 years and below. Males represented 46.9\% (70 405/150 213) of the study population and were noted to have a higher risk of PCR in comparison to females (95\% CI 1.04 to 1.17 ; OR: 1.11). The majority of subjects were Malays (42.1\%, 63 204/ $150213)$, followed by Chinese (33.8\%, 50758/150 213), Indians (13.9\%, 20 845/150 213) and other races (6.5\%, 9823/
150 213). No statistical difference was noted for the interethnicity PCR rate (table 2).

The most common ocular comorbidity was diabetic retinopathy which was present in 9.6\% (14 458/150 213) of study subjects. Diabetic retinopathy and pterygiums involving the cornea did not affect the PCR risk. The presence of pseudoexfoliation (95\% CI 1.02 to 1.82 ; OR: 1.36 ) and phacomorphic lens $(95 \%$ CI 1.25 to 3.06 ; OR: 1.96$)$ greatly increased the risk of PCR. Hypertension and diabetes were the most prevalent systemic illnesses, being noted in 58.6\% (88 094/150 213) and $42.0 \%$ (63 161/150 213) of study patients. respectively. The PCR risk was highest in diabetic (95\% CI 1.13 to 1.29 ; OR: 1.20$)$ and renal failure patients (95\% CI 1.09 to 1.55 ; OR: 1.30). Chronic obstructive airway disease/asthma did not increase the PCR risk.

Specialists performed most of the cataract procedures $(83.6 \%$, $125599 / 150$ 213). Surgeon seniority was noted to affect the risk of PCR where less experienced surgeons had an increased PCR rates (84\%-88\% higher risk). Majority of the surgeries were performed as day care procedures (51.6\%, 77 472/ 139 776). Admission type was not a risk factor for PCR. Phacoemulsification was the most common cataract procedure (82.3\%, 123 656/150 213). Subjects who underwent ECCE had less risk of PCR compared with those who had phacoemulsification cataract surgery (95\% CI 0.76 to 0.91 ; OR: 0.83$)$. Subjects with combined cataract surgery represented $1.9 \%$ (2850/ 150213 ) of the cataract procedures performed. Vitreoretinal surgery greatly increased the risk of intraoperative PCR $(95 \%$ CI 2.29 to 3.63 ; OR: 2.88 ).

The three most frequent intraoperative local anaesthesia given were topical $(51 \%, 76611 / 150213)$, followed by subtenon (37.0\%, $55644 / 150213)$ and intracameral (9.5\%, $14243 /$ $150213)$ anaesthesia. Facial block was the least preferred method of anaesthesia $(0.18 \%, 264 / 150213)$. Retrobulbar, subconjunctival and facial block anaesthesia did not statistically affect the surgical outcome. Subjects who had topical and intracameral anaesthesia were noted to have lower risk of PCR in comparison to peribulbar and subtenon anaesthesia.

\section{DISCUSSION}

Data from the study were contributed by $36 \mathrm{MOH}$ public hospitals, which represented the main providers of cataract service in Malaysia. The rise in cataract surgeries performed over the years was likely a result of expansion of ophthalmological services to rural areas, cataract outreach programmes and increased acceptance of phacoemulsification as the preferred surgical technique. Patient turnover was also higher with better surgeon acceptance

Table 1 Number of cataract patients and intraoperative complications for each year of the study

\begin{tabular}{|c|c|c|c|c|c|c|c|}
\hline Year & 2008 & 2009 & 2010 & 2011 & 2012 & 2013 & Total \\
\hline \multirow{2}{*}{ Patients, $\mathrm{n}$} & 21496 & 24438 & 28506 & 30611 & 32473 & 37150 & 174674 \\
\hline & n (\%) & n (\%) & n (\%) & n (\%) & n (\%) & n (\%) & n (\%) \\
\hline Any intraoperative complications & $1636(7.6)$ & $1645(6.7)$ & $1610(5.6)$ & $1787(5.8)$ & $1702(5.2)$ & $1998(5.4)$ & $10378(6.1)$ \\
\hline \multicolumn{8}{|l|}{ Types of complications } \\
\hline PCR & $798(3.7)$ & $858(3.5)$ & $840(2.9)$ & $936(3.1)$ & $870(2.7)$ & $1017(2.7)$ & $5319(3.1)$ \\
\hline Vitreous loss & $608(2.8)$ & $642(2.6)$ & $639(2.2)$ & $611(2.0)$ & $529(1.6)$ & $644(1.7)$ & $3673(2.2)$ \\
\hline Zonular dehiscence & $322(1.5)$ & $372(1.5)$ & $377(1.3)$ & $362(1.2)$ & $359(1.1)$ & $391(1.0)$ & $2183(1.3)$ \\
\hline Dropped nucleus & $33(0.2)$ & $40(0.2)$ & $38(0.1)$ & $58(0.2)$ & $56(0.2)$ & $63(0.2)$ & $288(0.2)$ \\
\hline Suprachoroidal haemorrhage & $10(0.0)$ & $13(0.1)$ & $9(0.0)$ & $8(0.0)$ & $8(0.0)$ & $8(0.0)$ & $56(0.0)$ \\
\hline Central corneal oedema & $27(0.1)$ & $22(0.1)$ & $26(0.1)$ & $36(0.1)$ & $30(0.1)$ & $23(0.1)$ & $164(0.1)$ \\
\hline Others & $361(1.7)$ & $373(1.5)$ & $338(1.2)$ & $449(1.5)$ & $439(1.3)$ & $572(1.5)$ & $2532(1.5)$ \\
\hline
\end{tabular}




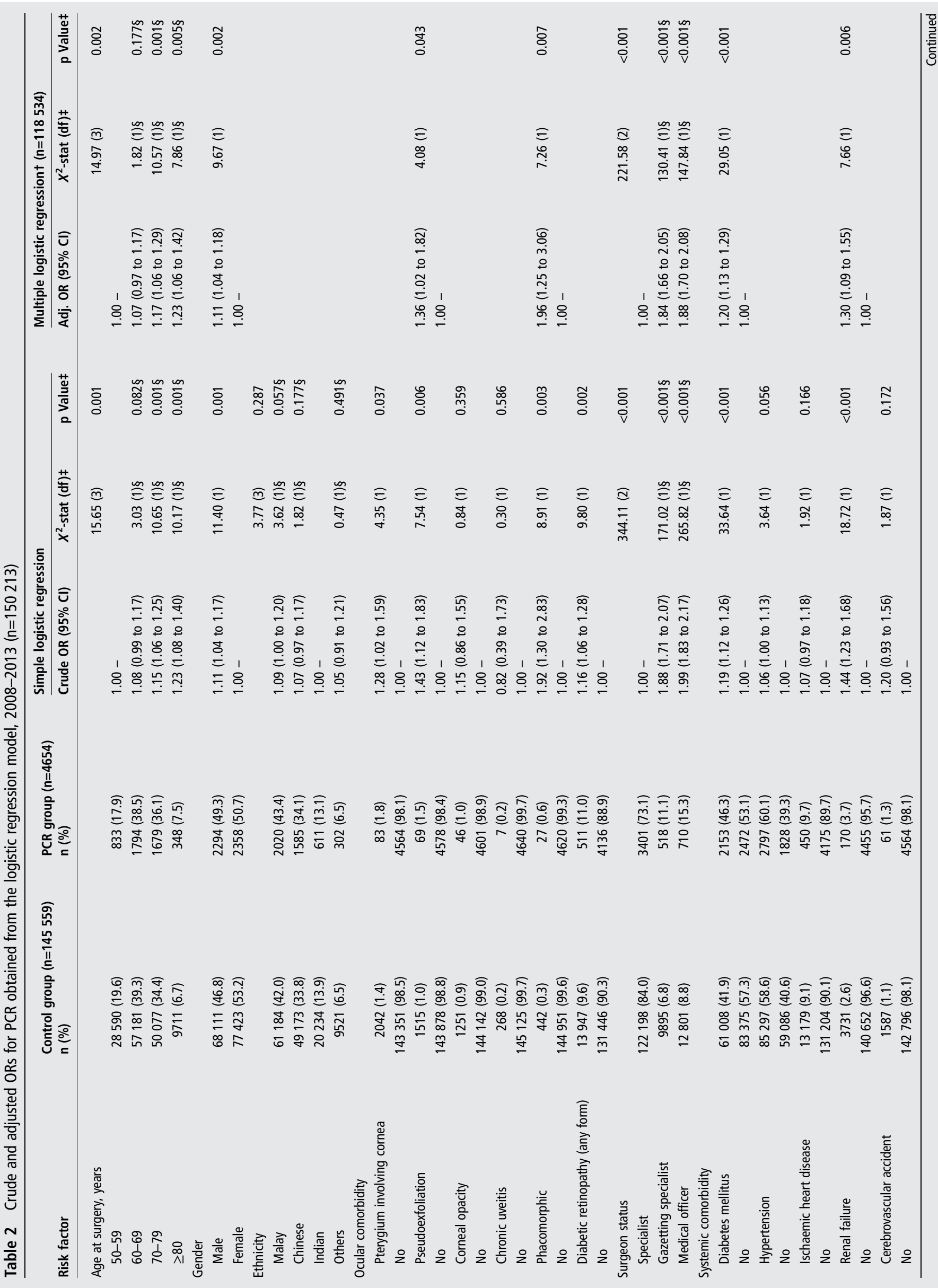




\section{Clinical science}

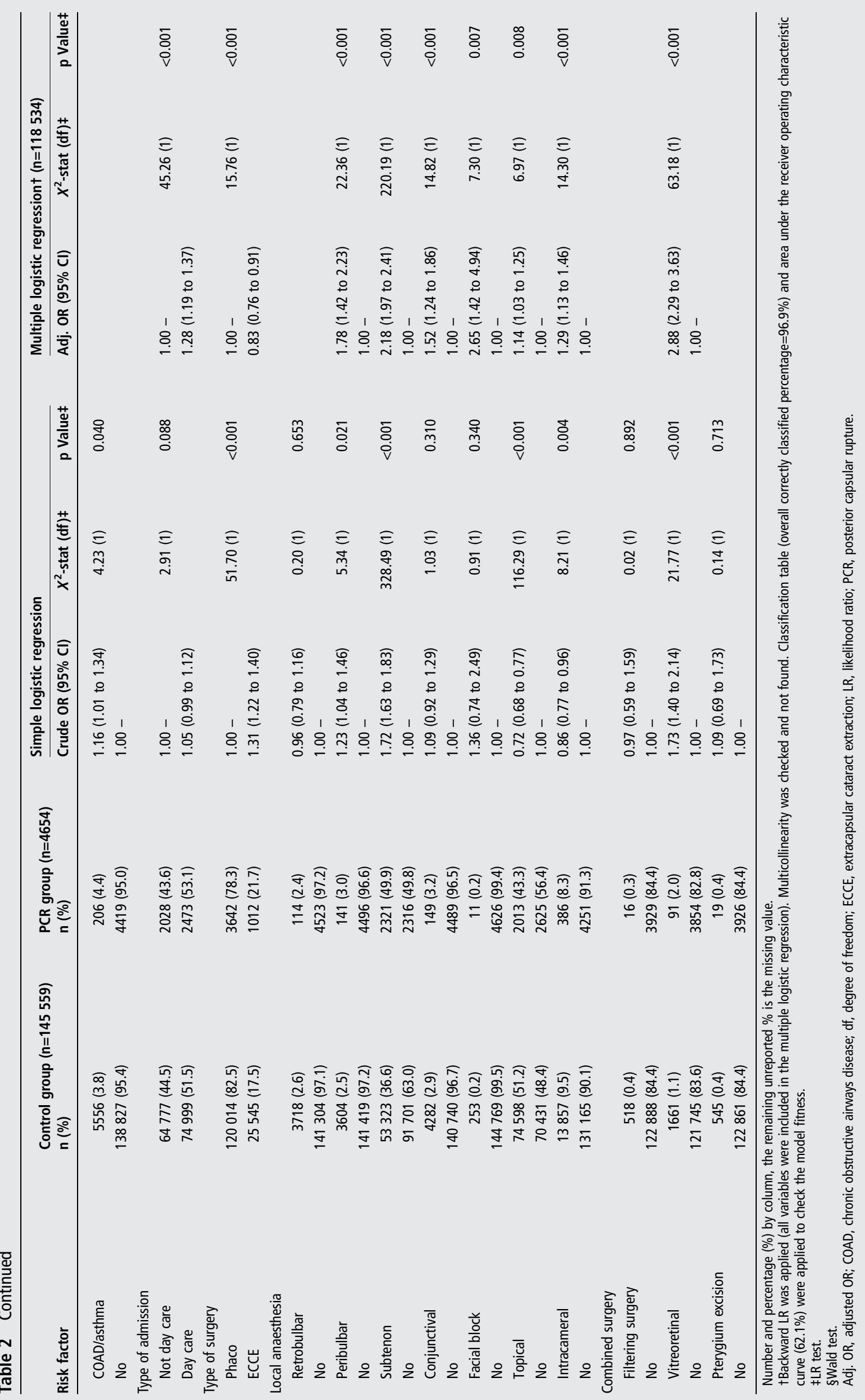


of intraoperative topical and intracameral anaesthesia and day care surgical facilities. ${ }^{9}$ Our PCR rate of 3.2\% was comparable to reported literature that reported PCR rates of $1.95 \%{ }^{11}-4.7 \% .^{12}$ The development of better surgical techniques and improved supervision of trainees may explain the reduction of intraoperative complication and PCR rate.

Our study findings of older age ( $\geq 70$ years) being a significant risk factor for PCR corresponded to other published studies. ${ }^{6} 13$ Older age may contribute to PCR due to weakening of the zonules with advancing age, increased lens density and other age-related ocular or systemic comorbidities that elevate the complexity of the cataract surgery performed. ${ }^{13}$ Our study noted no significant difference between the various ethnic groups. This finding was consistent with data from Singapore, which had a similar ethnic profile to Malaysia. ${ }^{14}$

Pseudoexfoliation and phacomorphic lens were significant PCR risk factors. Pseudoexfoliation is a well-known risk factor for PCR due to decreased pupil dilatation and weakened lens zonular support. The phacomophic lens is prone to increased intraoperative complications because of decreased corneal clarity from elevated intraocular pressure and the shallow anterior chamber from the intumescent lens, which impedes surgical instrumentation during cataract surgery.

Subjects with diabetes were noted to have more PCR. This was consistent with ocular changes in diabetes which potentially complicates cataract surgery such as decreased corneal clarity from diabetic keratopathy, impaired pupil dilatation and thickened lens capsule basement membrane which makes the lens capsule more friable and liable for rupture. ${ }^{15}$ Renal failure was also a significant PCR risk factor. This could be attributed to lens zonular stretching due to changes in anterior chamber depth from haemodialysis. ${ }^{16}$

Experience improves cataract surgery outcomes. ${ }^{17}$ This was noted that where senior surgeons had lower PCR rates compared with junior surgeons. We also noted cataract surgery combined with vitrectomy significantly increased PCR rates. This may be because vitrectomy causes fluctuation in anterior chamber depth, changes zonular support and affects posterior and anterior lens capsule stability. ${ }^{18}$

Anaesthesia type was reported not to affect the PCR rate. ${ }^{19}$ Our study however noted that kinetic anaesthesia such as topical and intracameral anaesthesia were associated with a lower risk of PCR compared with akinetic anaesthesia (retrobulbar, peribulbar and subtenon anaesthesia). This may be due to selection bias as the study was not randomised. Also, the patients who required akinetic anaesthesia may have had higher preoperative risk of PCR and akinetic anaesthesia were given in anticipation of complicated cataract surgery and prolonged surgery time.

We also noted that ECCE had a lesser risk of PCR. Our results may be a result of selection bias or could be due to surgeons increased familiarity with the ECCE technique as cataract surgeons in Malaysia are taught to master ECCE before they learn phacoemulsification.

The limitation of this study was that it was retrospective in nature. Also, as data were collected from a database, there would be a risk of under-reporting, lost data and selection bias. In addition, data entry by multiple individuals may have affected the accuracy of the data entered.

Our study however is still relevant as this was the largest multicentre survey of PCR for cataract surgery in Malaysia to date with good representation of all Malaysian ethnicities and fairly equal representation of both genders. In conclusion, this study is in agreement with other studies for the risk factors of PCR with the exception of local anaesthesia given and type of cataract surgery. We believe that with better identification of risk factors, the outcome of cataract surgery can be further refined for future minimisation of PCR risk.

Correction notice This article has been corrected since it was published Online First. The Acknowledgements section has been updated and a second affiliation has been added to the first author.

Acknowledgements The authors would like to thank the Director General of the Ministry of Health Malaysia for his kind permission to publish this article. The authors would also like to acknowledge the contribution of data entry by the Malaysian National Eye Database site coordinators, assigned optometrists and paramedical staff. The authors would also like to acknowledge the contribution of literature review and pre-drafting of the manuscript by Christopher James King (MA MB BChir) from Buckinghamshire Healthcare NHS Trust, United Kingdom.

Contributors Conception and design of the study (MAS, PPG, MI); analysis and interpretation (MAS, PPG, THA, FLMC, MI); writing of the article (MAS, PPG, THA, FLMC, MI); critical revision of the article (MAS, PPG, THA, FLMC, MI); final approval of the article (MAS, PPG, THA, FLMC, MI); data collection (MAS, PPG, MI); obtaining funding (MAS, PPG, MI); literature search (MAS, PPG, FLMC): administrative, technical or logistic support (MAS, PPG, THA, FLCM, MI).

Funding This study was supported by a grant from the Malaysian Ministry of Health Research Grant.

Competing interests None declared.

Ethics approval Ethical approval was obtained from the Medical Research and Ethics Committee of the Malaysian Ministry of Health.

Provenance and peer review Not commissioned; externally peer reviewed

\section{REFERENCES}

1 Ho J, Claoué C. Cataract skills: how do we judge competency? J R Soc Med 2013;106:2-4.

2 Salowi MA, Choong YF, Goh PP, et al. CUSUM: a dynamic tool for monitoring competency in cataract surgery performance. Br J Ophthalmol 2010;94:445-9.

3 Eng $Y Y$, Wee $\mathrm{JH}$. Visual outcome and complications after posterior capsule rupture during phacoemulsification surgery. Int Ophthalmol 2000;23:57-60.

4 Narendran $\mathrm{N}$, Jaycock P, Johnston RL, et al. The Cataract National Dataset electronic multicentre audit of 55567 operations: risk stratification for posterior capsule rupture and vitreous loss. Eye (Lond) 2009;23:31-7.

5 Muhtaseb M, Kalhoro A, Ionides A. A system for preoperative stratification of cataract patients according to risk of intraoperative complications: a prospective analysis of 1441 cases. Br J Ophthalmol 2004;88:1242-6.

6 Greenberg PB, Tseng VL, Wu WC, et al. Prevalence and Predictors of Ocular Complications Associated with Cataract Surgery in United States Veterans. Ophthalmology 2011;118:507-14.

7 Lundström M, Behndig A, Kugelberg M, et al. Decreasing rate of capsule complications in cataract surgery. Eight-year study of incidence, risk factors, and data validity by the Swedish National Cataract Register. J Cataract Refr Surg 2011;37:1762-7.

8 Chen M, LaMattina KC, Patrianakos T, et al. Complication rate of posterior capsule rupture with vitreous loss during phacoemulsification at a Hawaiian cataract surgical center: a clinical audit. Clin Ophthalmol 2014;8:375-8.

9 Lee MY, Goh PP, Salowi MA, et al. The Malaysian cataract surgery registry: cataract surgery practice pattern. Asia Pac J Ophthalmol 2014;3:343-7.

10 Goh PP, Elias H, Norfariza N, et al. National eye database - a web based surveillance system. Med J Malaysia 2008;63:20-3.

11 Day AC, Donachie PHJ, Sparrow JM, et al. The Royal College of Ophthalmologists National Ophthalmology Database study of cataract surgery: report 1, visual outcomes and complications. Eye (Lond) 2015;29:552-60.

12 Tsinopoulos IT, Karras GI, Haidich AB, et al. Association between age and incidence of posterior capsule rupture during cataract surgery: Cross-sectional study. J Cataract Refract Surg 2015;41:1783-4.

13 Hashemi H, Rezvan F, Etemad K, et al. Intraoperative complications of cataract surgery in Tehran Province, Iran. Optom Vis Sci 2016;93:266-71.

14 Chan FM, Mathur R, Ku JJK, et al. Rates of posterior capsule rupture during cataract surgery among different races in Singapore. Ann Acad Med Singap 2006;35:698-700.

15 Javadi MA, Zarei-Ghanavati $S$. Cataracts in diabetic patients: a review article. J Ophthalmic Vis Res 2008;3:52-65.

16 Wong CW, Wong TY, Cheng CY, et al. Kidney and eye diseases: common risk factors, etiological mechanisms, and pathways. Kidney Int 2014;85:1290-302.

17 Ti SE, Yang YN, Lang SS, et al. A 5-year audit of cataract surgery outcomes after posterior capsule rupture and risk factors affecting visual acuity. Am J Ophthalmol 2014; 157:180-5

18 Jalil A, Steeples L, Subramani S, et al. Microincision cataract surgery combined with vitrectomy: a case series. Eye (Lond) 2014;28:386-9.

19 Lee RMH, Foot B, Eke T. Posterior capsule rupture rate with akinetic and kinetic block anesthetic techniques. J Cataract Refract Surg 2013;39:128-31. 